\title{
Current management options for recurrent adrenocortical carcinoma
}

This article was published in the following Dove Press journal:

OncoTargets and Therapy

5 June 2013

Number of times this article has been viewed

\author{
Anthony R Glover' \\ Julian CY IP' \\ Jing Ting Zhao' \\ Patsy S H Soon ${ }^{1,4}$ \\ Bruce G Robinson ${ }^{1,3}$ \\ Stan B Sidhu' ${ }^{1,2}$ \\ 'Kolling Institute of Medical Research, \\ Cancer Genetics Laboratory, \\ ${ }^{2}$ Endocrine Surgical Unit, ${ }^{3}$ Department \\ of Endocrinology, Royal North Shore \\ Hospital and University of Sydney, \\ St Leonards, ${ }^{4}$ Department of Surgery, \\ Bankstown Hospital and University \\ of New South Wales, Bankstown, \\ NSW, Australia
}

Correspondence: Stan B Sidhu Suite 202, Level 2, 69 Christie Street, St Leonards, NSW 2065, Australia Tel +6I 29437 |73|

Email stansidhu@nebsc.com.au

\begin{abstract}
Adrenal cortical carcinoma (ACC) is a rare cancer that poses a number of management challenges due to the limited number of effective systemic treatments. Complete surgical resection offers the best chance of long-term survival. However, despite complete resection, ACC is associated with high recurrence rates. This review will discuss the management of recurrent ACC in adults following complete surgical resection. Management should take place in a specialist center and treatment decisions must consider the individual tumor biology of each case of recurrence. Given the fact that ACC commonly recurs, management to prevent recurrence should be considered from initial diagnosis with the use of adjuvant mitotane. Close follow up with clinical examination and imaging is important for early detection of recurrent disease. Locoregional recurrence may be isolated, and repeat surgical resection should be considered along with mitotane. The use of radiotherapy in ACC remains controversial. Systemic recurrence most often involves liver, pulmonary, and bone metastasis and is usually managed with mitotane, with or without combination chemotherapy. There is a limited role for surgical resection in systemic recurrence in selected patients. In all patients with recurrent disease, control of excessive hormone production is an important part of management. Despite intensive management of recurrent ACC, treatment failure is common and the use of clinical trials and novel treatment is an important part of management.
\end{abstract}

Keywords: recurrence, surgery, chemotherapy, mitotane, treatment

\section{Background}

Adrenal cortical carcinoma (ACC) has an incidence of approximately $0.5-2.0 /$ million per year. ${ }^{1-3}$ ACC can develop at any age with a maximal incidence seen in adults aged 40-50 years. ${ }^{4}$ Clinical presentation is due to functional symptoms related to excess adrenal hormone production, local symptoms related to tumor mass/local invasion, or incidental diagnosis by radiology. ACC is a heterogeneous disease characterized by a generally poor prognosis with an overall 5-year survival of less than $35 \%{ }^{5,6} \mathrm{At}$ presentation, most patients have localized disease amenable to surgical resection, and $30 \%-40 \%$ of patients will have systemic disease. ${ }^{6-8}$ Due to poor response rates to systemic treatments, few patients with systemic disease achieve complete remission. Complete surgical resection offers the best hope for long-term survival; however ACC remains a challenging malignancy as many patients develop recurrent disease despite complete resection. In isolated cases, patients with systemic disease will have remission following treatment. Although it is not a focus of this review, the management principles for the treatment of recurrence following systemic remission are similar to recurrence following complete surgical resection. 


\section{Pathology}

A challenge in the management of ACC is to quantify the recurrence risk from diagnosis. In the absence of metastatic disease or local invasion, the pathological diagnosis of ACC alone can be difficult due to the lack of clear-cut morphological criteria. ${ }^{9}$ Differential diagnostic scores have been developed for pathological diagnosis; currently the Weiss and modified Weiss scores are the most widely accepted scoring systems and are based on histological findings. ${ }^{10,11}$ These systems are not infallible and tumors initially diagnosed as benign can have unpredictable behavior. Immunohistochemistry provides important information for the diagnosis of ACC. ${ }^{12}$ Insulin-like growth factor 2 (IGF-2) overexpression has been identified in up to $90 \%$ of patients with sporadic ACC compared to adrenal cortical adenomas. ${ }^{13}$ The proliferation marker Ki-67 has also been demonstrated to be of importance in determining ACC and a patient's risk of recurrence. A high Ki-67 index ( $>10 \%)$ is associated with a shortened diseasefree status and decreased overall survival., ${ }^{9,14}$

Experimentally, the use of transcriptome, microRNA, and methylation profiling have been useful in diagnosing ACC, and offer the potential to stratify the risk of recurrence; however these techniques are yet to be validated in clinical practice. ${ }^{15-19}$

One example of this experimentation is the gene expression analysis of 153 adrenal tumors by de Reynies et al, where adrenal tumors were able to be classified into distinct diagnostic and prognostic groups. ${ }^{20}$ This study found differential expression of the cell cycle regulation genes DLG7 and PINK1 were a reliable indicator of benign and malignant tumors. ACCs were able to be classified into two distinct prognostic groups based on the combined expression of the genes $B U B 1 B$ and PINK1, with the better prognostic group less likely to have recurrent disease following surgery and also having an improved overall survival rate.

\section{Management following initial surgical resection}

Due to the high risk of recurrent disease, management of recurrence should be considered from the initial diagnosis. As with other malignancies, the management should take place in a multidisciplinary setting. Following surgery, the surgical resection margins need to be carefully assessed. If a complete resection (R0) has not been achieved, the role and feasibility of further surgical resection needs to be considered, as complete surgical resection is a critical prognostic factor. ${ }^{1,7}$ Patients with functional tumors should have post-operative hormone levels assessed as these levels can be used to indicate the completeness of surgical resection. ${ }^{21}$

\section{Adjuvant mitotane}

Adjuvant mitotane needs to be considered in all patients following surgical resection. Mitotane, a synthetic derivative of the insecticide dichlorodiphenyltrichloroethane (DDT) is an adrenolytic agent that has been the mainstay of medical management for metastatic ACC since it was discovered in the 1950s. However, its role in the adjuvant setting is less clear. ${ }^{5}$ Adjuvant mitotane has been shown in a number of retrospective case control series to improve recurrencefree survival following complete surgical resection; $;^{22,23}$ but these results have been criticized due to the possibility of selection bias and confounding. ${ }^{24}$ Adjuvant mitotane requires long-term treatment and frequent drug monitoring. ${ }^{25}$ Toxicity such as anorexia, nausea, vomiting, and diarrhea are common and can limit adjuvant mitotane's use as a long-term treatment. ${ }^{1,24,25}$ Mitotane use is associated with hypertriglyceridemia, changes in hormone binding globulins, and induction of hepatic microsomal enzymes leading to multiple drug interactions. ${ }^{3,25}$ These effects, and the need for monitoring, are further discussed in the section of mitotane therapy for systemic recurrence.

It has been suggested that, due to the difficulties associated with long term mitotane therapy, the use of adjuvant mitotane can be determined by the risk of recurrence, which can be stratified into low to intermediate and high risk. ${ }^{26}$ Patients with a high recurrence risk are defined by an initial tumor size of $>8 \mathrm{~cm}$, microscopic invasion of blood vessels/tumor capsule, or a Ki-67 index of $>10 \%$. These patients should be offered adjuvant mitotane routinely and considered for radiotherapy to the adrenal bed. Patients without these indications can be classified as low to intermediate risk, and for these patients the Efficacy of Adjuvant Mitotane Treatment (ADIUVO) study is currently recruiting. ${ }^{27}$ This study is an international randomized trial comparing mitotane to observational follow up. Until these results are available most centers recommend adjuvant mitotane after complete resection. ${ }^{1}$

\section{Follow up after surgical resection}

Due to the risk of recurrence and the possibility of further surgical treatment for recurrent ACC patients, close follow up is required. Early detection of recurrence is important as local recurrence or limited metastatic disease may be amenable to complete surgical resection (R0), which can be followed by long periods of disease-free survival. ${ }^{1}$ 
Follow-up should take the form of clinical assessment and progress imaging by computerized tomography (CT) with or without positron emission tomography (PET) scanning. In specialist centers, a range of follow up protocols has been suggested. These protocols include abdominal and chest CT every 3 months for the first 2 years post-surgery, increasing to 6-12 monthly intervals for at least 5 years. ${ }^{21,25}$ PET scans can be used as an alternative to CT imaging. ${ }^{28}$ Other imaging such as bone scans should be performed on a symptomatic basis. Patients with functional tumors should have hormone levels measured every 3 months as a means of early detection of tumor recurrence. ${ }^{21}$ A novel follow-up method currently under investigation is the use of urine steroid metabolomics, which the EURINE-ACT study is currently assessing as a technique to detect recurrence in comparison to imaging. ${ }^{29}$

\section{Recurrence rates and the role of specialist centers}

ACC recurs commonly after complete surgical resection with reported rates of $21 \%-91 \%{ }^{7,22,23,30,31}$ The wide ranges of reported recurrences are likely due to variability with patient selection of each series. Most series reporting recurrence are retrospective, including patients who have been referred to specialist centers after the development of recurrence, thereby creating a selection bias. This was highlighted by the series of Fassnacht et al which compared Stage II ACC patients, who were followed up prospectively following surgery by a specialist unit, against a group of patients referred 4 months or more after surgery. ${ }^{23}$ Patients referred at the time of initial surgery were found to have a recurrence rate of $30 \%$, compared to $74 \%$ for patients referred later. This series also found improved 5-year survival rates of $96 \%$ in patients referred initially, compared to $55 \%$ in patients referred later. In this series, patients who were referred at an earlier point of time were more likely to receive adjuvant mitotane, and it is thought the survival benefit could be due to a combination of patient selection and the increased use of mitotane. There is additional evidence that treatment in a high-volume center (defined by number of ACC cases treated each year, with 0.8 indicating a high volume center) may also improve treatment and survival. ${ }^{32}$

If available, treatment in a specialist center is important. ACC is rare, and this low incidence makes it difficult for individual medical centers to gain experience in its treatment. The advent of specialist centers has allowed for the development of international ACC study collaborations and led to prospective trials, such as the First International Randomized trial in locally advanced and Metastatic
Adrenocortical Carcinoma Treatment (FIRM-ACT) trial. ${ }^{53}$ These collaborative efforts are leading to a greater knowledge of the natural history and management of ACC and improving treatment.

ACC can recur at any time, with most recurrences occurring in the first 2 years following surgery, with $40 \%$ of patients relapsing in this time. ${ }^{31}$ After complete resection, more than $50 \%$ of patients will develop recurrence within 5 years. ${ }^{8,33}$

Locoregional recurrence comprises $19 \%-60 \%$ of total recurrences reported. ${ }^{34-36}$

Approximately $25 \%$ of patients have isolated locoregional recurrence. ${ }^{34}$ Locoregional recurrence can involve the pancreas, spleen, liver, diaphragm, and retroperitoneum. ${ }^{37}$

Systemic recurrences are most commonly detected in the lungs and liver with reported rates of $39 \%-65 \%$ total recurrences. ${ }^{7,35,37}$ Around $25 \%$ of systemic recurrences have been reported in lymph nodes, and $15 \%$ reported in bone. ${ }^{37}$

\section{Management of recurrent ACC}

When deciding on management, it is essential to consider the underlying tumor biology of ACC for each individual patient. ACC is an aggressive malignancy with a varied and heterogeneous natural history, and its treatment, such as extensive surgery, can carry considerable morbidity. Tumor biology is assessed by clinical judgment; it has been suggested that a minimum recurrence-free period of 6-12 months should be used to select patients who are likely to benefit from further surgery. ${ }^{26,38}$ Patients who have developed recurrence within 3 months of surgical resection, have rapidly progressive symptoms, or a lactate dehydrogenase level of $>1000$ units/L indicate rapidly progressive $\mathrm{ACC}$ and are unlikely to respond to current treatment. ${ }^{1}$ The management of recurrent disease will be discussed for locoregional (adrenal bed) and systemic disease recurrence.

\section{Locoregional recurrence Surgery}

With isolated locoregional recurrence, repeat surgery in selected patients can lead to improved survival. ${ }^{31,33}$ An aggressive surgical approach to achieve complete resection (R0) is advisable in patients who are fit for reoperation. ${ }^{34}$ In a recent large series review, Datrice et al have shown that resection for recurrent disease is safe and of benefit in patients with a disease-free interval of greater than 12 months. ${ }^{38}$ The surgical principles during reoperation are the same as those during the index procedure, with the aim to completely resect 
all disease, as incomplete resection leads to poorer survival outcomes. $^{7}$

If complete resection is not possible, debulking of tumors offers little benefit, but may be considered in selected patients prior to adjuvant therapy. ${ }^{21}$ Debulking surgery is of most benefit in patients with functional tumors not controlled by medical treatment.

For patients with isolated locoregional recurrence not amenable to a likely $\mathrm{R} 0$ resection, neo-adjuvant combined chemotherapy should be offered with the aim of complete resection if there is a response to treatment.

Surgery should be undertaken in a specialist center by an experienced endocrine surgeon in consultation with a multidisciplinary team. ${ }^{27}$ An open surgical approach is recommended for reoperation for locoregional recurrence due to the risk of tumor spillage, while minimally invasive surgery is inappropriate in this setting. ${ }^{39}$

\section{Radiotherapy}

Radiotherapy remains a controversial subject in the treatment of ACC and has traditionally been reserved for situations of palliative therapy. ${ }^{40}$ There has been limited evidence and no clear consensus guidelines in the specific role of neo-adjuvant or adjuvant radiotherapy. ${ }^{21}$ The proximity of the adrenal bed to sensitive organs such as the kidney, liver, spinal cord, and small bowel has made treatment difficult, especially with older radiotherapy techniques. ${ }^{41}$

Despite this, radiotherapy should be considered in patients with locoregional recurrence. Most evidence for radiotherapy is in regards to adjuvant radiotherapy. The evidence is conflicting; some series have demonstrated that patients who received adjuvant radiotherapy to the resection bed were less likely to develop locoregional recurrence, ${ }^{35}$ while other specialist centers have found no difference with the use of adjuvant radiotherapy. ${ }^{42}$ Guidelines proposed by the German ACC consortium recommend commencement of adjuvant radiotherapy within 3 months of surgery for patients with microscopically involved or inderterminate resection margins and stage III disease regardless of resection status. In addition, radiotherapy should be considered for tumors greater than $8 \mathrm{~cm}$, with a Ki-67 Index $>10 \%$ or invasion of adjacent vasculature. ${ }^{43}$

In a retrospective series over a 20 year period of 58 patients with 64 treatment instances of ACC, it was found that a 4.7 times higher risk of local recurrence was associated with a lack of radiotherapy, compared to treatment regimens that involved radiotherapy after surgical resection; however there was no difference in overall survival. ${ }^{44}$ This study had a number of limitations including a mix of treatment episodes, with 37 treatments for primary ACC and 27 for recurrent disease. Treatments involved surgery alone in 38 instances; ten instances were for surgery with adjuvant radiotherapy, and 16 for definitive radiotherapy for unresectable disease.

Radiotherapy has been shown to be effective in a palliative setting, especially to symptomatic bone, brain, and inferior vena cava disease. ${ }^{41,43,45}$

Given the conflicting evidence, the role of radiotherapy is yet to be elucidated by multicenter prospective trials. Until this data is available, radiotherapy should be considered after reoperation for locoregional control and for definitive treatment in recurrence not amenable to surgical resection.

\section{Radiofrequency ablation}

Percutaneous image-guided radiofrequency ablation (RFA) has appeal in delivering a minimally invasive, locally effective treatment, especially in patients who are not candidates for reoperation.

Available evidence has shown that RFA can produce effective, short-term local control of primary ACC in an adjuvant setting for tumors less than $50 \mathrm{~mm}$ in size, that are not near sensitive tissues or large blood vessels. ${ }^{46}$ RFA has been demonstrated in a small case series of $15 \mathrm{ACC}$ recurrences to be well tolerated, with $53 \%$ of patients demonstrating decrease in tumor size or loss of enhancement on imaging. ${ }^{46}$ Smaller tumors fared better $(<50 \mathrm{~mm}$ ), with up to $67 \%$ demonstrating complete ablation.

RFA in combination with surgical resection may allow for better disease control in the setting of local and isolated systemic recurrence. ${ }^{38}$ However, side effects of bleeding, infection, and injury to adjacent organs can occur. Nevertheless, with advancing technology, RFA has the potential to have a role in treatment options of recurrent ACC in selected patients. $^{38}$

\section{Systemic recurrence}

Patients who develop systemic disease have a poor prognosis, with a 5-year survival rate of less than $15 \%$. $622,31,47$ Conventional medical treatment is mitotane, with or without chemotherapy, and adrenal hormone control. However, response rates to medical treatment remain disappointing. ${ }^{3}$ There is a limited role for surgical resection in a small number of selected patients. Given the limited treatment options and poor prognosis of ACC, novel treatments and 
clinical trials are important components in the treatment of systemic disease.

\section{Medical treatment}

Conventional or first line treatments can be mitotane alone or mitotane in combination with chemotherapy. European Society for Medical Oncology guidelines recommend combination chemotherapy and mitotane for patients with inoperable ACC with high tumor volume and rapid disease, whilst mitotane alone can be used initially for patients with low tumor volume, slow progression, or those unfit for chemotherapy. ${ }^{3}$

\section{Mitotane}

Patients who have not previously received systemic treatment should receive high dose mitotane as a first line treatment. ${ }^{1}$ High dose mitotane aims for a blood level of 14-20 mg/L. Mitotane blood levels should be checked following 3 weeks of treatment, and if plasma levels remain low $(<7 \mathrm{mg} / \mathrm{L})$, the dose should be adjusted and cytotoxic chemotherapy considered. Supportive treatment with hydrocortisone substitution is required to cover adrenal insufficiency, and fludrocortisone may be required depending on blood pressure, serum potassium levels, and plasma renin activity. ${ }^{3}$ The effect of mitotane to induct cytochrome P450 3A4 means that up to half of administered hydrocortisone can be rapidly inactivated, requiring high steroid doses to obtain adequate cover. ${ }^{48}$ Mitotane inhibits $5 \alpha$-reductase and androgen replacement may also be required. ${ }^{48}$ Liver function tests, thyroid function, and blood lipid levels need to be monitored while patients are receiving mitotane. ${ }^{3}$

Approximately one third of patients will receive a response but generally there is little increase in survival rates, ${ }^{25,49,50}$ although there are reported isolated cases of longterm disease control ${ }^{51}$ and rare cases of disease remission. ${ }^{52}$ For functional tumors, an additional benefit of mitotane is that it will control hormone excess in most patients. ${ }^{50}$

\section{Chemotherapy plus mitotane}

Patients who have rapidly progressing disease should receive combination treatment as a first line treatment; however, the response rates are poor, with few patients receiving long term benefit. ${ }^{1}$ Chemotherapy used in ACC as conventional treatment includes either streptozocin, or the combination etoposide, doxorubicin, and cisplatin (EDP). These two treatments were compared in the FIRM-ACT study. ${ }^{53}$ A response rate of $23 \%$ was found in the EDP-mitotane group, versus $9 \%$ in the streptozocin-mitotane group. The
EDP-mitotane group had a 5 month progression-free survival advantage compared to 2 months for the Streptozocinmitotane group, but no significant overall survival benefit. Despite the poor response, this trial is important as it is the largest prospective trial (304 patients) undertaken on ACC and provides a baseline against which further novel treatments can be compared. Given these results, patients should receive EDP and mitotane as a first line combination treatment. ${ }^{5}$

Patients receiving mitotane with or without chemotherapy should be assessed at two-monthly intervals for tumor progression. Patients who show tumor regression or stable disease should be considered for surgical resection or continuation of therapy. Patients with progressive disease should consider other chemotherapy regimens or be enrolled in a clinical trial.

\section{Salvage chemotherapy treatments}

There are currently no established second or third line chemotherapy regimens for systemic disease. ${ }^{54}$ However, a phase-II trial of gemcitabine plus capecitabine has shown some encouraging results, with $46 \%$ of patients having stable disease for more than 4 months, ${ }^{55}$ and this has been suggested as a possible second/third line regimen. ${ }^{1}$ Patients should be considered for clinical trials if they have progressive disease with conventional treatment.

\section{Medical treatment of steroid excess}

Patients with systemic disease must undergo monitoring and control of steroid hormone excess, as they can die from infections and hypercortisolism, rather than from tumor burden. This is especially important in the setting of chemotherapyinduced immunosuppression. ${ }^{28}$ Adrenal enzyme inhibition can be achieved with the use of mitotane, ketoconazole, metyrapone, etomidate, or mifepristone. These medications require close monitoring and frequent dose adjustments. ${ }^{56}$

\section{Surgery and other local treatments}

Surgical resection has a role in selected patients with systemic recurrence of ACC. Patients with metastatic disease amenable to complete surgical resection, for example liver or lung metastasis, should be considered for resection even if it is required to be completed over two stages. ${ }^{21,57,58}$ Complete surgical resection of recurrent disease in a medically fit and symptomatic patient can be of benefit and is associated with long-term survival in some patients. ${ }^{7,59}$ Any decision in offering radical surgical treatment is dependent on the tumor biology and will not offer any benefit for patients with rapidly progressive tumors. ${ }^{38}$ 
The role for debulking surgery in systemic ACC is more limited and should only be considered for patients with uncontrollable hormone production. In this situation, debulking surgery can provide effective symptom palliation. ${ }^{1,7}$

Radiotherapy and other ablation techniques (RFA/laser) also have a limited role in systemic recurrence but can be useful for palliation for local symptomatic and hormone production control in selected cases. ${ }^{43,60}$

A suggested plan of management for recurrent ACC is shown in Figure 1.

\section{Novel and future treatments}

The poor survival results with systemic ACC demonstrates the need for improved treatment options, and a number of novel and targeted therapies have been studied with mixed results as discussed below.

\section{Tyrosine kinase inhibitors}

Treatments trialled have included targeted treatments against epidermal growth factor receptor (EGFR).
EGFR is overexpressed in most ACCs, making it a useful target for treatment; ${ }^{61}$ however, targeted treatment with the tyrosine kinase inhibitor erlotinib plus gemcitabine showed a limited response as a salvage treatment, with a response only seen in one out of ten patients. ${ }^{62}$ Poor response was also seen with the use of imatinib. ${ }^{63}$ Sunitinib, which targets several tyrosine kinase inhibitors, has been shown to have a modest response, with some patients achieving stable disease in a phase-II trial. ${ }^{64}$ This trial showed worse results with concomitant mitotane, possibly due to reduced serum sunitinib levels caused by mitotane induced cytochrome P450-3A4 activity.

More promising results have been seen with the targeting of insulin-like growth factor 1 receptor (IGF-1R). IGF2, which is over-expressed in ACCs, is known to signal predominantly through IGF-1R. ${ }^{65}$ Study of the oral tyrosine kinase inhibitor (OSI-906) which targets IGF-1R has shown promising results, with stabilization of disease seen in five out of 16 patients. ${ }^{6}$ This agent is currently being evaluated by the GALACCTIC clinical trial. ${ }^{67}$

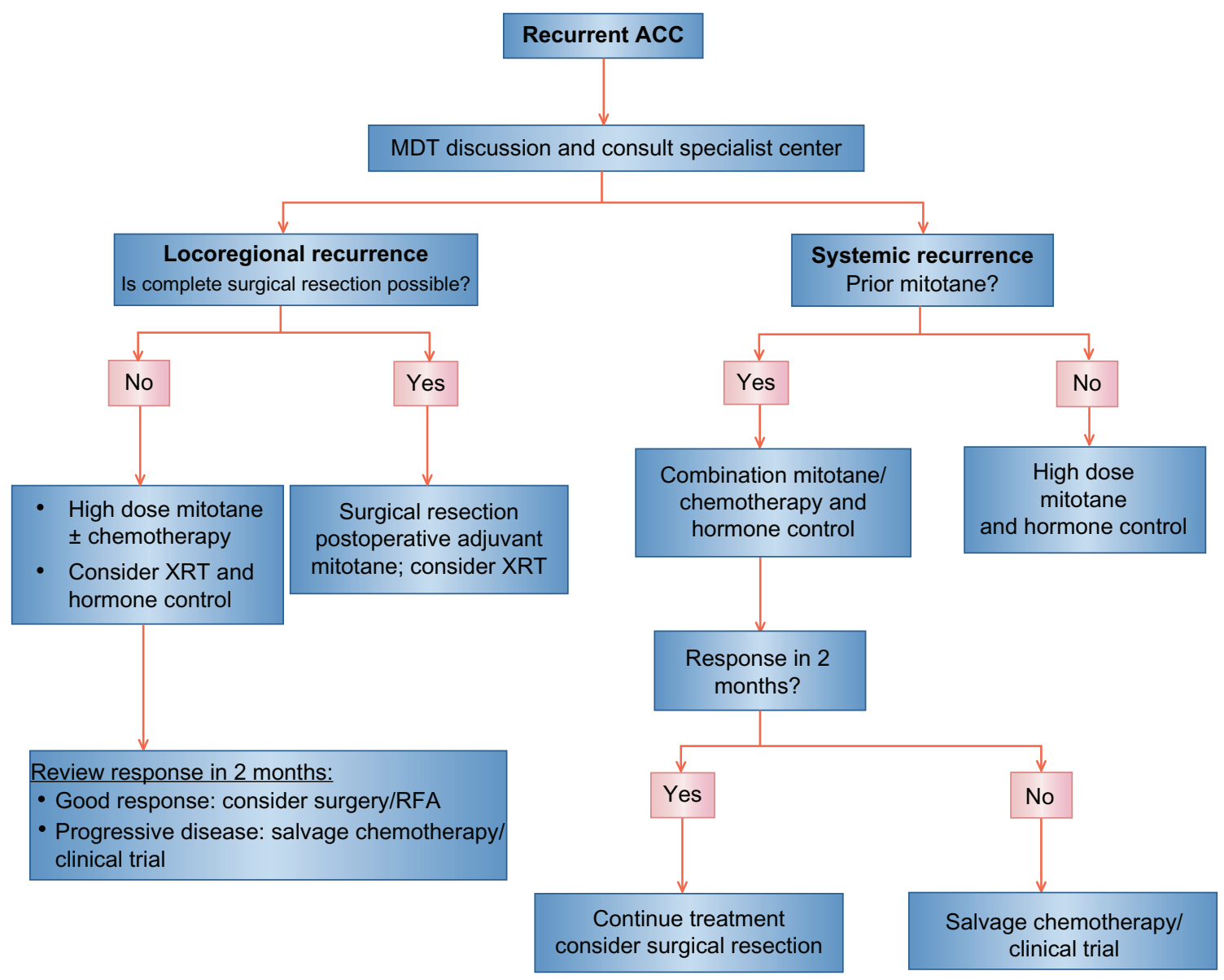

Figure I Suggested management plan for recurrent disease.

Abbreviations: ACC, Adrenal cortical carcinoma; MDT, multidisciplinary team; RFA, radio-frequency ablation; XRT, radiotherapy. 


\section{Angiogenesis inhibitors}

Due to the highly vascular nature of ACC, vascular endothelial growth factor (VEGF) has also been assessed as a treatment target; however, a study of bevacizumab used in combination with capecitabine showed no response in ten patients as a salvage treatment, with severe toxicity seen in two patients. ${ }^{68}$

\section{Mammalian target of rapamycin signaling}

Another target of interest in ACC is mammalian target of rapamycin (mTOR) due to its relationship with IGF-1R. mTOR is a downstream signaling node for a number of receptor tyrosine kinases including IGF-1R; however, inhibition of mTOR alone leads to activation of other tumorigenic pathways, thereby limiting its use as a single agent for the treatment of malignancy. ${ }^{69}$ An exploratory study of the use of everolimus, a rapamycin analog, has shown no clinical response. ${ }^{70}$ Combination treatment targeting mTOR showed more promise in a recent phase-I trial using the IGF-1R antibody cixutumumab combined with temsirolimus, where $42 \%$ of patients achieved stable disease for a minimum of 6 months. ${ }^{71}$

\section{Future treatments}

Other targets of interest include the $\mathrm{Wnt} / \beta$-catenin pathway, interleukin-13 receptor alpha2, microRNA therapies, and demethylating agents. ${ }^{19,72-74}$

\section{Conclusion}

Recurrence of ACC remains a difficult disease to treat, but with a multidisciplinary approach it is possible for some patients to achieve long-term disease control and survival. As ACC is a rare disease, many patients are only referred to specialist centers following the development of recurrence. To achieve better outcomes, patients should be referred to, or advice sought from, a specialist center at the initial suspicion of ACC. The poor response rates to current systemic treatment offer ACC as a model to researchers to identify new treatment targets and assess novel treatments to improve outcomes in this difficult disease.

\section{Disclosure}

The authors report no conflicts of interest in this work.

\section{References}

1. Fassnacht M, Libé R, Kroiss M, Allolio B, Medscape. Adrenocortical carcinoma: a clinician's update. Nat Rev Endocrinol. 2011;7(6):323-335.

2. Wajchenberg BL, Albergaria Pereira MA, Medonca BB, et al. Adrenocortical Carcinoma: clinical and laboratory observations. Cancer. 2000;88(4):711-736.
3. Berruti A, Baudin E, Gelderblom H, et al. Adrenal cancer: ESMO Clinical Practice Guidelines for diagnosis, treatment and follow-up. Ann of Oncol. 2012;23(Suppl 7):vii131-vii138.

4. Kebebew E, Reiff E, Duh QY, Clark OH, McMillan A. Extent of disease at presentation and outcome for adrenocortical carcinoma: have we made progress? World J Surg. 2006;30(5):872-878.

5. Kirschner LS. The next generation of therapies for adrenocortical cancers. Trends in Endocrinol Metab. 2012;23(7):343-350.

6. Abiven G, Coste J, Groussin L, et al. Clinical and biological features in the prognosis of adrenocortical cancer: poor outcome of cortisolsecreting tumors in a series of 202 consecutive patients. J Clin Endocrinol Metab. 2006;91(7):2650-2655.

7. Schulick RD, Brennan MF. Long-term survival after complete resection and repeat resection in patients with adrenocortical carcinoma. Ann Surg Oncol. 1999;6(8):719-726.

8. Stojadinovic A, Ghossein RA, Hoos A, et al. Adrenocortical carcinoma: clinical, morphologic, and molecular characterization. J Clin Oncol. 2002;20(4):941-950.

9. Fassnacht M,Allolio B. Clinical management of adrenocortical carcinoma Best Pract Res Clin Endocrinol Metab. 2009;23(2):273-289.

10. Lau SK, Weiss LM. The Weiss system for evaluating adrenocortical neoplasms: 25 years later. Hum Pathol. 2009;40(6):757-768.

11. Aubert S, Wacrenier A, Leroy X, et al. Weiss system revisited: a clinicopathologic and immunohistochemical study of 49 adrenocortical tumors. Am J Surg Pathol. 2002;26(12):1612-1619.

12. Soon PSH, Gill AJ, Benn DE, et al. Microarray gene expression and immunohistochemistry analyses of adrenocortical tumors identify IGF2 and Ki-67 as useful in differentiating carcinomas from adenomas. Endocr Relat Cancer. 2009;16(2):573-583.

13. Schmitt AA, Saremaslani PP, Schmid SS, et al. Rearrangements at the $11 \mathrm{p} 15$ locus and overexpression of insulin-like growth factor-II gene in sporadic adrenocortical tumors. J Clin Endocrinol Metab. 1994; 78(6):1444-1453.

14. Morimoto R, Satoh F, Murakami O, et al. Immunohistochemistry of a proliferation marker Ki67/MIB1 in adrenocortical carcinomas: Ki67/ MIB1 labeling index is a predictor for recurrence of adrenocortical carcinomas. Endocr J. 2008;55(1):49-55. Available at: http://eutils. ncbi.nlm.nih.gov/entrez/eutils/elink.fcgi?dbfrom=pubmed\&id=18187 873\&retmode $=$ ref\&cmd=prlinks. Accessed January 14, 2013.

15. Gicquel CC, Bertagna XX, Gaston VV, et al. Molecular markers and long-term recurrences in a large cohort of patients with sporadic adrenocortical tumors. Cancer Res. 2001;61(18):6762-6767.

16. Assie G, Giordano TJ, Bertherat J. Gene expresion profiling in adrenocortical neoplasia. Mol Cell Endocrinol. 2012;351(1):111-117. Available at: http://www.sciencedirect.com/science/article/pii/ S0303720711006162. Accessed February 14, 2013.

17. Soon PS, Libé R, Benn DE, et al. Loss of heterozygosity of 17p13, with possible involvement of ACADVL and ALOX15B, in the pathogenesis of adrenocortical tumors. Ann Surg. 2008;247(1):157-164.

18. Patterson EE, Holloway AK, Weng J, Fojo T, Kebebew E. MicroRNA profiling of adrenocortical tumors reveals miR-483 as a marker of malignancy. Cancer. 2010;117(8):1630-1639.

19. Rechache NS, Wang Y, Stevenson HS, et al. DNA methylation profiling identifies global methylation differences and markers of adrenocortical tumors. J Clin Endocrinol Metab. 2012;97(6):E1004-E1013.

20. de Reynies A, Assie G, Rickman DS, et al. Gene expression profiling reveals a new classification of adrenocortical tumors and identifies molecular predictors of malignancy and survival. J Clin Oncol. 2009; 27(7):1108-1115.

21. Schteingart DE, Doherty GM, Gauger PG, et al. Management of patients with adrenal cancer: recommendations of an international consensus conference. Endocr Relat Cancer. 2005;12(3):667-680.

22. Terzolo M, Angeli A, Fassnacht M, et al. Adjuvant mitotane treatment for adrenocortical carcinoma. N Engl J Med. 2007;356(23):2372-2380.

23. Fassnacht M, Johanssen S, Fenske W, et al. Improved survival in patients with stage II adrenocortical carcinoma followed up prospectively by specialized centers. J Clin Endocrinol Metab. 2010;95(11):4925-4932. 
24. Huang H, Fojo T. Adjuvant mitotane for adrenocortical cancer - a recurring controversy. J Clin Endocrinol Metab. 2008;93(10):3730-3732.

25. Allolio B, Fassnacht M. Adrenocortical carcinoma: clinical update. J Clin Endocrinol Metab. 2006;91(6):2027-2037.

26. Fassnacht M, Allolio B. What is the best approach to an apparently nonmetastatic adrenocortical carcinoma? Clin Endocrinol. 2010;73(5):561-565.

27. University of Turin, Italy. Efficacy of Adjuvant Mitotane Treatment (ADIUVO). In: ClinicalTrials.gov [website on the Internet]. Bethesda, MD: US National Library of Medicine; 2008 [updated 2011]. Available from: http://clinicaltrials.gov/show/NCT00777244. NLM identifier: NCT00777244. Accessed January 10, 2013.

28. Lacroix A. Approach to the patient with adrenocortical carcinoma. J Clin Endocrinol Metab. 2010;95(11):4812-4822.

29. Arlt W, Biehl M, Taylor AE, et al. Urine steroid metabolomics as a biomarker tool for detecting malignancy in adrenal tumors. J Clin Endocrinol Metab. 2011;96(12):3775-3784.

30. Kendrick ML, Lloyd R, Erickson L, et al. Adrenocortical carcinoma: surgical progress or status quo? Arch Surg. 2001;136:543-549.

31. Crucitti F, Bellantone R, Ferrante A, Boscherini M, Crucitti P, Group TAIRS. The Italian registry for Adrenal Cortical Carcinoma: analysis of a multiinstitutional series of 129 patients. The ACC Italian Registry Study Group. Surgery. 1996;119:161-170.

32. Lombardi CP, Raffaelli M, Boniardi M, et al. Adrenocortical carcinoma: effect of hospital volume on patient outcome. Langenbecks Arch Surg. 2011;397(2):201-207.

33. Pommier RF, Brennan MF. An eleven-year experience with adrenocortical carcinoma. Surgery. 1992;112:963-971.

34. Bellantone R, Ferrante A, Boscheriui M, et al. Role of reoperation in recurrence of adrenal cortical carcinoma: Results from 188 cases collected in the Italian National Registry for Adrenal Cortical Carcinoma. Surgery. 1997;122(6):1212-1218.

35. Fassnacht M, Hahner S, Polat B, et al. Efficacy of adjuvant radiotherapy of the tumor bed on local recurrence of adrenocortical carcinoma. J Clin Endocrinol Metab. 2006;91(11):4501-4504.

36. Lombardi CP, Raffaelli M, De Crea C, et al. Open versus endoscopic adrenalectomy in the treatment of localized (stage I/II) adrenocortical carcinoma: results of a multiinstitutional Italian survey. Surgery. 2012;152(6):1158-1164.

37. Brennan MF. Adrenocortical Carcinoma. CA Cancer J Clin. 1987; 37(6):349-365.

38. Datrice NM, Langan RC, Ripley RT, et al. Operative management for recurrent and metastatic adrenocortical carcinoma. J Surg Oncol. 2011;105(7):709-713.

39. Miller BS, Gauger PG, Hammer GD, Doherty GM. Resection of adrenocortical carcinoma is less complete and local recurrence occurs sooner and more often after laparoscopic adrenalectomy than after open adrenalectomy. Surgery. 2012;152(6):1150-1157.

40. Percarpio B, Knowlton AH. Radiation therapy of adrenal cortical carcinoma. Acta Radiol Ther Phys Biol. 1976;15(4):288-292.

41. Lebastchi AH, Kunstman JW, Carling T. Adrenocortical Carcinoma: Current Therapeutic State-Of-The-Art. J Oncol. 2012;2012:1-11.

42. Habra MA, Ejaz S, Feng L, et al. A Retrospective cohort analysis of the efficacy of adjuvant radiotherapy after primary surgical resection in patients with adrenocortical carcinoma. J Clin Endocrinol Metab. 2013;98(1):192-197.

43. Polat B, Fassnacht M, Pfreundner L, et al. Radiotherapy in adrenocortical carcinoma. Cancer. 2009;115(13):2816-2823.

44. Sabolch A, Feng M, Griffith K, Hammer G, Doherty G, Ben-Josef E. Adjuvant and Definitive Radiotherapy for Adrenocortical Carcinoma. Int J Radiat Oncol Biol Phys. 2011;80(5):1477-1484.

45. Milgrom SA, Goodman KA. The role of radiation therapy in the management of adrenal carcinoma and adrenal metastases. J Surg Oncol. 2012;106(5):647-650.

46. Wood BJ, Abraham J, Hvizda JL, Alexander HR, Fojo T. Radiofrequency ablation of adrenal tumors and adrenocortical carcinoma metastases. Cancer. 2003;97(3):554-560.
47. Icard P, Goudet P, Charpenay C, et al. Adrenocortical carcinomas: surgical trends and results of a 253-patient series from the French Association of Endocrine Surgeons study group. World J Surg. 2001;25(7):891-897.

48. Chortis V, Taylor AE, Schneider P, et al. Mitotane therapy in adrenocortical cancer induces CYP3A4 and inhibits $5 \alpha$-reductase, explaining the need for personalized glucocorticoid and androgen replacement. J Clin Endocrinol Metab. 2013;98(1):161-171.

49. Haak HR, Hermans J, van deVelde CJH, et al. Optimal treatment of adrenocortical carcinoma with mitotane: results in a consecutive series of 96 patients. Br J Cancer. 1994;69(5):947-951.

50. Hahner S, Fassnacht M. Mitotane for adrenocortical carcinoma treatment. Curr Opin Investig Drugs. 2005;6(4):386-394.

51. Kornely E, Schlaghecke R. Complete remission of metastasized adrenocortical carcinoma under o,p'-DDD. Exp Clin Endocrinol. 1994;102(1):50-53.

52. Ilias I, Alevizaki M, Philippou G, Anastasiou E, Souvatzoglou A. Sustained remission of metastatic adrenal carcinoma during long follow up. J Endocrinol Invest. 2001;24(7):532-535

53. Fassnacht M, Terzolo M, Allolio B, et al. Combination chemotherapy in advanced adrenocortical carcinoma. $N$ Engl J Med. 2012;366(23):2189-2197

54. Fassnacht M, Kreissl MC, Weismann D, Allolio B. New targets and therapeutic approaches for endocrine malignancies. Pharmacol Ther. 2009; 123(1):117-141.

55. Sperone P, Ferrero A, Daffara F, et al. Gemcitabine plus metronomic 5-fluorouracil or capecitabine as a second-/third-line chemotherapy in advanced adrenocortical carcinoma: a multicenter phase II study. Endocr Relat Cancer. 2010;17(2):445-453

56. Veytsman I, Nieman L, Fojo T. Management of Endocrine Manifestations and the Use of Mitotane As a Chemotherapeutic Agent for Adrenocortical Carcinoma. J Clin Oncol. 2009;27(27):4619-4629.

57. Gaujoux S, Al-Ahmadie H, Allen PJ, et al. Resection of Adrenocortical Carcinoma Liver Metastasis: Is it Justified? Ann Surg Oncol. 2012;19(8):2643-2651.

58. Winkel den JO, Pfannschmidt J, Muley T, et al. Metastatic adrenocortical carcinoma: results of 56 pulmonary metastasectomies in 24 patients. Ann Thorarc Surg. 2011;92(6):1965-1970.

59. Mihai R, Iacobone M, Makay O, et al. Outcome of operation in patients with adrenocortical cancer invading the inferior vena cava - a European Society of Endocrine Surgeons (ESES) survey. Langenbecks Arch Surg. 2011;397(2):225-231.

60. Pacella CM, Stasi R, Bizzarri G, et al. Percutaneous laser ablation of unresectable primary and metastatic adrenocortical carcinoma. Eur $J$ Radiol. 2008;66(1):88-94.

61. Adam P, Hahner S, Hartmann M, et al. Epidermal growth factor receptor in adrenocortical tumors: analysis of gene sequence, protein expression and correlation with clinical outcome. Mod Pathol. 2010;23(12): $1596-1604$

62. Quinkler M, Hahner S, Wortmann S, et al. Treatment of advanced adrenocortical carcinoma with erlotinib plus gemcitabine. JClin Endocrinol Metab. 2008;93(6):2057-2062.

63. Gross DJ, Munter G, Bitan M, et al. The role of imatinib mesylate (Glivec) for treatment of patients with malignant endocrine tumors positive for c-kit or PDGF-R. Endocr Relat Cancer. 2006;13(2):535-540.

64. Kroiss M, Quinkler M, Johanssen S, et al. Sunitinib in refractory adrenocortical carcinoma: a phase II, single-arm, open-label trial. J Clin Endocrinol Metabolism. 2012;97(10):3495-3503.

65. Tacon LJ, Prichard RS, Soon PS, Robinson BG, Clifton-Bligh RJ, Sidhu SB. Current and Emerging Therapies for AdvancedAdrenocortical Carcinoma. Oncologist. 2011;16(1):36-48.

66. Macaulay VM, Middleton MR, Eckhardt SG, et al. Phase I study of OSI-906, dual tyrosine kinase inhibitor of insulin-like growth factor-1 receptor (IGF-1R) and insulin receptor (IR) in combination with erlotinib (E) in patients with advanced solid tumors. J Clin Oncol. 2010;28(15):3016. 
67. Astellas Pharma Inc. A Study of OSI-906 in Patients With Locally Advanced or Metastatic Adrenocortical Carcinoma (GALACCTIC). In: ClinicalTrials.gov [website on the Internet]. Bethesda, MD: US National Library of Medicine; 2009 [updated 2012]. Available from http:// clinicaltrials.gov/show/NCT00924989. NLM identifier NCT00924989. Accessed January 10, 2013.

68. Wortmann S, Quinkler M, Ritter C, et al. Bevacizumab plus capecitabine as a salvage therapy in advanced adrenocortical carcinoma. Eur J Endocrinol. 2010;162(2):349-356.

69. Memmott RM, Dennis PA. Akt-dependent and -independent mechanisms of mTOR regulation in cancer. Cell Signal. 2009;21(5):656-664.

70. Fraenkel M, Gueorguiev M, Barak D, Salmon A, Grossman AB, Gross DJ. Everolimus therapy for progressive adrenocortical cancer. Endocrine. Epub February 16, 2013.
71. Naing A, LoRusso P, Fu S, et al. Insulin growth factor receptor (IGF-1R) antibody cixutumumab combined with the mTOR inhibitor temsirolimus in patients with metastatic adrenocortical carcinoma. $\mathrm{Br} J$ Cancer. 2013;108(4):826-830.

72. Tissier F, Cavard C, Groussin L, et al. Mutations of beta-catenin in adrenocortical tumors: activation of the wnt signaling pathway is a frequent event in both benign and malignant adrenocortical tumors. Cancer Res. 2005;65(17):7622-7627.

73. Iorio MV, Croce CM. MicroRNA dysregulation in cancer: diagnostics, monitoring and therapeutics. A comprehensive review. EMBO Mol Med. 2012;4(3):143-159.

74. Jain M, Zhang L, He M, et al. Interleukin-13 receptor alpha2 is a novel therapeutic target for human adrenocortical carcinoma. Cancer. 2012; 118(22):5698-5708.
OncoTargets and Therapy

\section{Publish your work in this journal}

OncoTargets and Therapy is an international, peer-reviewed, open access journal focusing on the pathological basis of all cancers, potential targets for therapy and treatment protocols employed to improve the management of cancer patients. The journal also focuses on the impact of management programs and new therapeutic agents and protocols on

\section{Dovepress}

patient perspectives such as quality of life, adherence and satisfaction. The manuscript management system is completely online and includes a very quick and fair peer-review system, which is all easy to use. Visit http://www.dovepress.com/testimonials.php to read real quotes from published authors.

\footnotetext{
Submit your manuscript here: http://www.dovepress.com/oncotargets-and-therapy-journal
} 